\title{
O GESTO BIBLIOGRÁFICO E A MODERNIDADE
}

\section{GESTO BIBLIOGRÁFICO Y MODERNIDAD}

\author{
Vinícios Menezes - menezes.vinicios@gmail.com \\ Doutorando do Programa de Pós-graduação em Ciência da \\ Informação do Instituto Brasileiro de Informação em Ciência e \\ Tecnologia (IBICT).
}

\begin{abstract}
RESUMO
Introdução: A bibliografia partilha de uma esfera discursiva tensiva, ora intensamente textual, ora demasiadamente estatística. Por ora, em linhas gerais, põe-se a bibliografia em perspectiva como uma performance linguística que porta consigo milênios de tradição, reviravoltas, guinadas, estagnações, que todavia não lhe arranca o frescor. O inverso, a torna cada vez e sempre mais instigante, vide as falas de Gabriel Peignot "a bibliografia é o mais vasto e universal de todos os conhecimentos humano", e de Alfredo Serrai "a bibliografia é a mãe de todas as disciplinas da representação escrita".

Objetivo: Esse ensaio busca apresentar a dinâmica tensiva da Bibliografia e suas alternâncias valorativas. A intenção central é tecer como questão de princípio da Bibliografia a dimensão linguístico-pragmática.

Metodologia: $O$ enfoque teórico-reconstrutivo é o abordado, a partir das possibilidades de abertura às instâncias passíveis de desenvolvimento e realização. Entendendo a bibliografia como Paul Otlet, enquanto meio, guiamonos pelos seus usos - cognitivos e comunicativos. Contudo, também nos indagamos acerca da modernidade da bibliografia e suas questões de permanente discussão: tem por fim "algo maior" como uma ciência geral dos livros/documentos, como em Otlet? Ou, será arte exegética dos hermeneutas e críticos? Ou ainda, alguma coisa totalmente outra? Ou talvez, nem tanto nem tão pouco, há um equilíbrio reflexivo possível de ser estabelecido?

Resultados: Orientamo-nos "para" dimensão humanístico-universal implicada na bibliografia, acreditando ser esta um meio para o uso público da razão tão propagado, mas tão pouco realizado pela modernidade.

Conclusões: Ao final, são postos argumentos acerca de uma devida recontextualização e de uma possível reconstrução de alguns pressupostos bibliográficos, visando seus respectivos potenciais emancipatórios, outrora inconclusos no projeto do esclarecimento (Aufklärung).
\end{abstract}

Palavras-chave: Bibliografia. Filosofia da linguagem. Modernidade. 


\section{A BIBLIOGRAFIA EM SEU GESTO}

O pensador assemelha-se muito ao desenhador cujo objetivo é representar todas as inter-relações entre coisas $^{1}$ (WITTGENSTEIN, 2000, p. 27).

Vivemos em um tempo pretensamente sombrio para o biblion e uma das suas artes mais expressivas: a bibliografia. Birger Hjørland em seu texto Arguments for 'the bibliographical paradigm'. Some thoughts inspired by the new English edition of the UDC assinala que o paradigma bibliográfico tem sido omitido nos discursos da Biblioteconomia e Ciência da Informação (Library and Information Science - LIS). Quando mencionado, sua abordagem é, de uma maneira geral, pelo prisma da negatividade, por vezes sendo utilizado como contraste para "algo melhor", como em Carol Kuhlthau (2004) e o paradigma mentalista. Aos olhos de Hjørland, esta supressão do paradigma bibliográfico só leva à fragilização dos domínios Biblioteconomia e Ciência da Informação: "a ausência da perspectiva bibliográfica é também uma ausência de uma perspectiva humanista em documentos, gêneros e perspectivas históricas e culturais"², tendo em vista que está neste paradigma "crítico e pragmático" o seu fundamento justificador ${ }^{3}$. Essa ocultação discursiva demonstra um deficitário senso ético-moral para com os princípios intersubjetivos que outrora justificaram o campo enquanto legítimo - por sinal, nesse processo discursivo o paradigma bibliográfico foi um dos argumentos centrais de justificação. Ao que nos parece, e não nos cabe dizer muito mais além disso para não cairmos no oximoro temeroso do "abismo da superficialidade", a LIS baseia alguns dos seus pressupostos

\footnotetext{
1 Ao desenhador de Wittgenstein poderíamos sem sombra de dúvidas chamar o bibliógrafo. Talvez esta comparação wittgensteiniana tenha como pano de fundo a crítica platônica às artes pictóricas, sendo aqui o pensador um sujeito da mimese e não das ideias. Assim, o bibliógrafo, como o pensador, objetivam representar todas as inter-relações entre coisas.

2 "The absence of the bibliographical perspective is also an absence of a humanist perspective on documents, genres and historical and cultural perspectives." (HJØRLAND, 2007)

${ }^{3}$ A respeito da "quase ausência" do paradigma bibliográfico no discurso da LIS, Hjørland (2007) apresenta este como "tudo o que o campo é", nós não partilhamos desta totalização, em vista da multidiversidade das relações que se sobrepõem histórico e socialmente ao campo da LIS. Todavia, estamos concordes que o paradigma bibliográfico é vital para o entendimento da LIS.
} 
epistemológicos num modelo conversionista das ciências naturais, constrangedor de um conjunto argumentativo teórico-prático validado e socialmente estabilizado em detrimento de outro emergente, capaz de resolver problemas não solucionáveis, recém surgidos ou não observados pelo anterior. Logo, trata-se de conversão, não de (re)construção ou associação - ainda que esses dois últimos não sejam de todo excludentes à conversão. Nesta querela entre tradição e inovação, ficamos com Wittgenstein (2000, p. 65):

Deves dizer algo de novo e, contudo, tudo o que dizes deve ser velho. De fato, deves limitar-te a dizer coisas velhas - e, não obstante, o que dizes deve ser algo de novo! Diferentes interpretações devem corresponder a diferentes aplicações.

O título do texto chama-se "o gesto bibliográfico" com uma intenção: apontar para a dimensão linguístico-pragmática da bibliografia. Stephen Paling (2004, p. 588, grifo nosso) nos diz: "A Bibliografia fornece uma vantagem convincente para estudar a interligação de classificação, retórica e construção do conhecimento." E continua, "A Bibliografia [...] tem relação direta com os estudos textuais e a retórica." Nesta mesma direção, Donald Mackenzie afirma que "a bibliografia é uma disciplina que estuda a sociologia dos textos", ou ainda, para evidenciar uma perspectiva reconciliadora podemos reinterpretar a concepção de Buonocore (1976, p. 67): "a bibliografia é erudição e técnica ao mesmo tempo, vale dizer, uma disciplina essencialmente pragmática." Com isto tenta-se reconciliar e agregar ao gesto bibliográfico as estruturas (princípio organizador) cognitivas das coisas, da práxis do social e da expressão dos sujeitos. De muitos modos esses mundos são acessíveis para a bibliografia. No conjunto das falas do evento "A arte da Bibliografia" é nítida uma expressão pungente desses mundos conexos, que trataremos de associá-los à perfomatividade prática do fazer bibliográfico em seus usos cognitivos e comunicativos da linguagem. 
Designo por cognitivo o uso de atos de fala constativos em que têm sempre de figurar enunciados perlocutórios; aqui a relação interpessoal entre o locutor e o ouvinte, estabelecida de modo performativo, tem por objetivo o entendimento sobre objetos (ou estados de coisas). Já por comunicativo podemos designar 0 uso da linguagem em que, de forma inversa, o entendimento sobre objetos (ou estados de coisas) visa o estabelecimento de uma relação interpessoal. O plano da comunicação, que num dos casos representa o objetivo, no outro serve de meio. No uso cognitivo da linguagem, são tematizados conteúdos proposicionais; no uso comunicativo da linguagem, os conteúdos proposicionais são apenas referidos para gerar, de um modo performativo, uma determinada relação intersubjetiva entre locutores / ouvintes. A reflexividade das línguas naturais deve-se ao fato de ambos os modos de utilização da linguagem remeterem implicitamente um para o outro. (HABERMAS, 2010, p. 107)

Nestes termos, em contraste com a concepção por vezes unitária e positivista da "bibliografia física" (physical bibliography), nos associamos à perspectiva de Ranganathan, de uma "bibliografia social" (social bibliography), que não necessariamente nega a bibliografia física, mas a complementa em perspectiva. Em Ranganathan, a bibliografia social é o pano de fundo alicerçador e justificador das fundamentadoras cinco leis da biblioteconomia, o que nos leva à evidenciação da extrema importância da bibliografia no corpo teórico-epistêmico de Ranganathan, que por vezes não é projeto do corpo discursivo de análise da obra do bibliotecário indiano. A bibliografia, em Raganathan, está baseada no conceito relacional tripartido de livro, enquanto a) alma (tema), b) corpo sutil (assunto comunicado) e c) corpo grosseiro (fisicalidade). São essas as palavras de Ranganathan (1952, p. 23): "A book may, then, be taken to be a trinity of soul (=alma), subtle body (=sukshma sarira) and gross body (= sthula sarira). In association with each of these members of the trinity, the term, Bibliography' gets a different meaning."

Nor should it be as for analytical, or descriptive, or historical or critical or textual bibliography i.e. as for the scholars turned on the study of the books of the past as 
a foundation and a means to capture the details of the culture of the past. The new approach [social bibliography] should be towards the book of the future. It should follow the path of making books an effective tool in self-education and self-entertainment as much for the democracy as for the aristocracy (in the intellectual sense). (RANGANATHAN, 1952, p. 57)

Duas coisas são necessárias pontuar. A primeira é que a relação democracia e aristocracia no contexto de vida da Índia de Ranganathan é clara, sendo ele um parresiasta ao incluir em seu discurso a dimensão democrática do uso público da razão, face aos obstáculos do princípio organizativo indiano de poder. O segundo ponto é que por meio desta ideia de "self-education and self-entertainment" avistamos a relação entre a bibliografia e o mundo expressivo da personalidade.

No contexto brasileiro, possuímos a abordagem de Edson Nery da Fonseca (1979), ao bipolarizar de modo analítico a bibliografia em Bibliografia textual e Bibliografia estatística (bibliometria), sendo esta classificada em dois planos: a) bibliometria macrobibliográfica (bibliografias nacionais) e b) bibliometria microbibliográfica (cadeia de citações). O esforço de Edson Nery seria o de "tirar da bibliografia o seu aspecto de pura técnica ou mesmo de simples ciência auxiliar" ou desvincular o entendimento da bibliografia como "apenas uma lista de livros" (1979, p. 30). Três anos antes do texto de Edson Nery, Hagar Espanha Gomes em seu texto $\mathbf{O}$ ensino da bibliografia relatava a disparidade do ensino (e por ora, da conceituação) de Bibliografia no Brasil enquanto um problema epistêmico: "A falta de livro de texto [manual] em português parece ser a causa da disparidade de pontos de vista no ensino da Bibliografia" (1976, p. 93). Este problema ocupa boa parte das reflexões epistemológicas de Thomas Kuhn em sua obra $\mathbf{A}$ estrutura das revoluções científicas, especialmente quando diz: "Quando falo de fonte de autoridade, penso sobretudo nos principais manuais científicos, juntamente com os textos de divulgação e obras filosóficas moldadas naqueles" e mais adiante "Os próprios manuais pretendem comunicar o vocabulário e a sintaxe de uma linguagem 
científica" (2007, p. 176), pois o objetivo destes livros é "inevitavelmente persuasivo e pedagógico" (2007, p. 19). Desta maneira, Hagar Espanha aponta para esta lacuna de grande importância epistêmica descrita por Kuhn e avança quando complementa seu relato analítico de modo ainda mais crítico: "O que se ensina sob o tópico Bibliografia é 'o estudo dos repertórios' e não a problemática inerente à Bibliografia." (1976, p. 102) ${ }^{4}$. Portanto, a bibliografia no Brasil precisa desde muito ser revisitada.

Quando falamos de uma inter-relação entre a bibliografia e o estatuto cognitivo das coisas, temos em mente a clássica fundação da bibliografia, denominada na literatura por "bibliografia física" pela escola positivista, mas que reinterpretada a partir da turn linguist ganha uma fertilidade pela via da construção social de evidências. Assim, esta relação fica tão evidente quanto a epígrafe desse texto: a bibliografia mira a representação da inter-relação entre as coisas, por intermédio do ator "bibliógrafo", ausente na teoria clássica positivista. A dupla relação da bibliografia com a práxis social e a expressividade estética da personalidade, pode ser entendida nos usos comunicativos da linguagem. Vinculada a esta dúplice relação está a bibliografia social. $\mathrm{Na}$ conferência Nas bases da Bibliografia: reflexões sobre uma longa história, Giulia Crippa (2014) aponta para a bibliografia como um mapa, um plano de orientação para algo - em geral, o objeto de tematização delimitado a ser seguido. Deste modo, seguindo a asserção de Crippa, podemos estabelecer um nexo da "bibliografia social" com a "regra wittgensteiniana", que representa para a filosofia contemporânea uma das grandes contribuições para o entendimento pragmático do mundo. Em suas Investigações Filosóficas, Wittgenstein descreve o conceito de regra paulatinamente durante grande parte do livro; uma das primeiras incursões e a que nos chama inicialmente a atenção é: "uma regra está aí como uma placa de orientação" e, mais adiante, podendo

\footnotetext{
${ }^{4}$ Parece-me que aqui Hagar Espanha Gomes nos remete para a distinção entre bibliografia e catálogo. Para esclarecimentos acerca desta distinção consultar, Buonocore (1976, p. 34-36) e Paul Otlet (2007, p. 286-308).
} 
ser usado/lido no lugar de tabela a própria bibliografia, "a tabela é, portanto, uma regra pela qual ele se orienta ao executar uma ordem." (Wittgenstein, 2008, $\S 85-87$, p. 61-63) Crippa em sua fala trata com especial atenção da relação seminal entre Cassiodoro (aproximadamente 485-580 d.C.) e a bibliografia (mapa de orientação). Neste contexto relacional e conforme tal época, Giorgio Agamben (2013, 2014) faz uma análise do "nascimento" das regras em contraposição a Lex, sendo um multidiverso material bibliográfico, com especial atenção às regras monásticas, a fonte normatizadora das formas de vida (forma vitae) de então. Por exemplo, quando diz em Opus Dei acerca da aliança entre a "ação divina" e a "co-labor-ação dos homens": "Costumase atribuir, sobre a autoridade de Du Cange, a criação do sintagma opus Dei à regra beneditina, na qual este aparece algumas vezes para designar o ofício litúrgico" (AGAMBEN, 2013, p. 30). Enfim, a associação entre o uso comunicativo da linguagem e a bibliografia, tem um solo fértil de pesquisa em comunhão com a pragmática linguística wittgensteiniana. Com o propósito de deixar um pouco mais claro o que diz Wittgenstein a respeito da regra, ficamos com os "parágrafos" 201 e 202 das Investigações Filosóficas.

Nosso paradoxo era o seguinte: uma regra não poderia determinar um modo de agir, dado que todo modo de agir deve poder concordar com a regra. A resposta: se todo modo de agir deve poder concordar com a regra, então deve poder contradizê-la também. Por conseguinte, não haveria aqui nem concordância nem contradição. Que haja aqui um equívoco, mostra-se já no fato de que colocamos nesta ordem de ideias uma interpretação atrás da outra; como se cada interpretação nos tranquilizasse ao menos por um instante até pensarmos numa outra interpretação, que por sua vez está por trás desta. Com isso mostramos, a saber, que há uma concepção de regra que não é uma interpretação; mas que se exprime, de caso para caso da aplicação, naquilo que denominamos 'seguir a regra' e 'transgredi-la'. Por isso, existe uma tendência de dizer: todo agir de acordo com a regra é uma interpretação. No entanto, dever-se-ia denominar 'interpretar' somente: substituir uma expressão da regra por outra expressão. 
Por isso, 'seguir a regra' é uma prática. E acreditar seguir a regra não é: seguir a regra. $E$ por isso não se pode seguir a regra 'privatim', porque, do contrário, acreditar seguir a regra seria o mesmo que seguir a regra. (WITTGENSTEIN, 2008, § 201-202, p. 113-114).

Ao nos referirmos ao "gesto" acompanhamos as instruções de Walter Benjamin e Giorgio Agamben, onde o gesto é uma mimese primitiva da linguagem, no primeiro, e "palavra" ("gesto originário"), no segundo ${ }^{5}$. Conforme Agamben, o gesto é "puro meio", "comunicação de uma comunicabilidade"; todavia, esta "essência" da "comunicabilidade" não é ideal, prefigurada ou extramundana, mas, de acordo com as palavras Sérgio Rouanet (2008, p. 118): "a essência da ideia é linguística" e em complemento, afirma Wittgenstein (2008, § 434, p. 174): "o gesto tenta prefigurar - diríamos - mas não o pode." Em outras palavras, o gesto é linguístico-pragmático, fundado na cultura, na sociedade e na socialização entre pessoas. O que aqui estamos a chamar de gesto bibliográfico tem ancoragem em algumas conceituações práticas, em especial aquelas que apontam para a dimensão linguístico-pragmática da bibliografia. Vejamos o que diz Agamben sobre o gesto.

[...] não de imagem se deveria propriamente falar, mas de gestos. [...] O que caracteriza o gesto é que, nele, não se produz, nem se age, mas se assume e suporta. Isto é, o gesto abre a esfera do ethos como esfera mais própria do homem. [...] se o fazer é um meio em vista de um fim e a práxis é um fim sem meios, o gesto rompe a falsa alternativa entre fins e meios que paralisa a moral e apresenta meios que, como tais, se subtraem ao âmbito da medialidade, sem por isso tornarem-se fins. [...] O gesto é a exibição de uma medialidade, o tornar visível um meio como tal. Este faz aparecer o ser-num-meio do homem e, deste modo, abre para ele a dimensão ética. [...] assim, no gesto, é a esfera não de um fim em si, mas de uma medialidade pura e sem

\footnotetext{
5 Fazemos apenas uma ressalva à perspectiva agambeniana que por ter um propósito argumentativo rumo à subjetividade expressiva, não passa aos fins (práxis) - que em nossa perspectiva é o entendimento, mas que aos olhos de Agamben seria a obra (ergon).
} 
fim que se comunica aos homens. [...] Ela é, num meio, aquela potência do gesto que o interrompe no seu próprio ser-meio e apenas assim o exibe, faz de uma res uma res gesta. Do mesmo modo, compreendendose por palavra o meio da comunicação, mostrar uma palavra não significa dispor de um plano mais elevado (uma metalinguagem, esta mesma incomunicável no interior do primeiro nível), a partir do qual se faz dela objeto da comunicação, mas expô-la sem nenhuma transcendência na sua própria medialidade, no seu próprio ser meio. O gesto é, neste sentido, comunicação de uma comunicabilidade. Este não tem propriamente nada a dizer, porque aquilo que mostra é o ser-na-linguagem do homem como pura medialidade. [...] exposição do ser-na-linguagem do homem: gestualidade pura. [...] A política é a esfera dos puros meios, isto é, da absoluta e integral gestualidade dos homens. (AGAMBEN, 2008, p. 12-14).

Deste modo, transportados para as cercanias das teorias da ação, o ato de bibliografar, em Paul Otlet, é poiético, vem da poiésis hermêutico-retórica e indica um meio (por isso, também um gesto linguístico) para um fim. Logo, em concordância com o que foi dito, chegamos a Paul Otlet e lemos: "a bibliografia é um meio, não um fim" (OTLET, 2007, p. 286); o fim é a "bibliologia ou documentação" (OTLET, 2007, p. 9). O gesto bibliográfico em Otlet (2007, p. 288) é um meio, "nunca se acaba: deve continuar" e fazendo referência ao Eclesiastes 12:12 diz: "não há limite para fazer livros". A bibliografia é "a base de toda documentação" (OTLET, 2007, p. 286) um meio para um fim - a ciência geral do documento (bibliologia) baseada no "livro universal"6. "summa summarum", a "obra". Com esta definição Paul Otlet acaba por inconscientemente cercear o potencial emancipatório do seu projeto bibliográfico universal, fundado num dos pilares da modernidade, o uso público da razão. Otlet (2007, p. 429) suprime as possibilidades pragmáticas por ele promovidas diante de uma demasiada semantização do "livro universal", especialmente quando diz: "o

\footnotetext{
6 "Esto será, en pocas palabras, el libro universal (o el libro ideal, puro, sintético, íntegro, óptimo, futuro, anticipado)." (OTLET, 2007, p. 429).
} 
documento tipo do futuro deverá conter todas as possibilidades herdadas, junto com as que possa adquirir por si mesmo. Será uma síntese", uma totalidade absoluta. A bibliologia acaba se delineando como uma região, um conjunto artificial que agrega todas as possibilidades semânticas de descrição do mundo enquanto fato. Logo, o problema não está na dimensão universalista da bibliografia (não confundamos universalidade com totalidade ou fundamento último), mas no enrijecimento das possibilidades sociais de "descrição densa" da bibliologia, ou em outros termos, na supressão dos usos cognitivos (interobjetivos) e comunicativos (intersubjetivos) da linguagem.

\section{A BIBLIOGRAFIA E A MODERNIDADE (INACABADA)}

O livro está cheio de vida - não como um homem [privatum], mas como um formigueiro (WITTGENSTEIN, 2000, p. 95)

A relação entre universalidade, humanismo e bibliografia, levantada por Otlet em seu projeto, pode ser alongada por outras vias, quiçá frente à "another universalism", como sugestionado pelo projeto crítico reconstrutivo de Seyla Benhabib (2011). Autores clássicos como Gabriel Peignot, Paul Otlet, Mudge, Calot, Thomas, ou, contemporâneos como Tennis e Buckland, denominam a bibliografia como uma ciência descritiva do biblion/documento. Joseph Tennis em seu artigo Is there a new bibliography? apresenta esta como "enumeração sistemática e descritiva de escritos - 'obras (meta-escritos), textos (autores) e artefatos (reprodução)"' (2011, p. 123); Buckland (1992) em seu Redesigning Library Services: A Manifest tipifica a bibliografia em enumerativa, descritiva e crítica. O que fica claro é o papel representativo da linguagem na bibliografia, em particular a "descrição gramatical", isto é, a descrição das nossas formas de representação através dos usos cognitivos da linguagem orientados à construção social de evidências. É neste sentido, de uma gramática profunda dos usos gerais da linguagem que podemos reconstruir o projeto humanista 
universal de Otlet, em especial quando diz que o "progresso intelectual geral" dependerá primeiro de uma "língua mais simples, mais potente, mais geral" (OTLET, 2007, p. 430), ou seja, da linguagem ordinária do mundo das vivências intersubjetivas e linguisticamente estruturadas. $O$ gesto bibliográfico funda-se nos "universais pragmáticos" da linguagem, consideravelmente nos "dêiticos" que apontam para o que os modernos chamavam, de um modo geral, de signatura rerum (assinatura das coisas) - vide, por exemplo, a expressão utilizada por Otlet para a representação documentária: signatura (colocação).

$\mathrm{Na}$ via prática da "biblioteca sem muros" - para usar uma expressão de Roger Chartier (1999) - Otlet é um moderno; assim como quando Gabriel Naudé (1627) aponta para o uso público da razão implícito no "ideal de biblioteca" quando diz: "nenhum meio mais honesto e seguro para adquirir um grande renome entre os povos do que construir belas e magníficas bibliotecas, para depois consagrá-las à utilidade pública"; ou ainda, na inovação do uso da palavra biblioteca efetuado pelo polímeta Conrad Gesner (Bibliotheca Universalis), investindo a este o signo universal da bibliografia ("biblioteca sem parede") imbricado no livro-mundo da modernidade. Logo, ao chegarmos ao final do século XVIII e início do XIX nos deparamos com Gabriel Peignot e a sua definição de bibliografia: "a bibliografia é o mais vasto e universal de todos os conhecimentos humanos, tudo parece ter que ser da responsabilidade do bibliógrafo" (1802, p. 50, tradução nossa). André Vieira de Freitas Araújo (2014), em sua palestra Pionerismo bibliográfico em um polímeta do séc. XVI: Conrad Gesner no evento A Arte da Bibliografia, argumentou junto às proposições de Alfredo Serrai que "a bibliografia é a mãe de todas as disciplinas da representação escrita". O reflexo desse conceito está em Otlet ao afirmar que a bibliografia foi a primeira a apresentar no "livro" o problema da universalidade, ultrapassando, nas palavras do humanista belga, a questão da biblioteca e suas coleções particulares; manifestase Otlet: "a bibliografia tem introduzido assim em toda a documentação 
o espírito universal e enciclopédico". (2007, p. 255). O ideal universalista e humanista das Luzes ganha nesses bibliólogos sua expressão e associa-se a figuras comuns da modernidade, como Kant e o processo de universalização como "ato puro de entendimento" (entender-se com alguém sobre algo), Voltaire e o "fundo comum da humanidade", Rousseau e o cosmopolitismo da "grande cidade mundo" ou, o contemporâneo Habermas e seu projeto reconstrutivo da ação comunicativa de uma modernidade inacabada.

Portanto, ao final pontuo em reticências a dimensão linguísticopragmática como ancoragem da bibliografia em seu projeto humanísticouniversal; à diferença de boa parte dos modernos, entendemos que o humano já é, de saída, social; antropologicamente, é da condição humana ser dotada de linguagem - o mais universal dos atributos da espécie. Na voz de poeta, nos encanta Mia Couto (2013, p. 7): "a pessoa é uma humanidade individual". Seguindo o ensinamento de Karl Jaspers (importa ser de seu próprio tempo), vejo-nos confrontados não mais apenas com o mundo físico, que centralmente atravessou nossos interlocutores e em especial Otlet; todavia, nos encontramos também, para usar o termo de Capurro, como seres-num-mundo-digital; por conseguinte, em nossa "luta com a linguagem" trata-se de uma tarefa ética ser distributivo. O gesto bibliográfico para ser universal deve ser "qualquer", nem particular nem total; o ser do projeto humanistauniversal é um ser-qualquer. Se a linguagem (ou livro em Otlet, Mallarmé, Borges, Rendon Rojas) é a casa do ser, aqui, na bibliografia, nos encontramos em casa.

\footnotetext{
${ }^{7}$ Para exemplos da pólis associada ao biblion, vejam a Bibliópolis de Otlet (2007, p. 419-420) e o texto de Giulia Crippa $O$ patrimônio cultural: a cidade como documento.
} 


\section{AGRADECIMENTOS}

Agradeço aos organizadores do evento A Arte da Bibliografia pelo convite, a confiança e a solicitude da partilha de um tema tão valioso para a tradição crítica sociocultural do ocidente.

\section{REFERÊNCIAS}

AGAMBEN, Giorgio. Altíssima pobreza: regras monásticas e forma de vida. São Paulo: Boitempo, 2014.

AGAMBEN, Giorgio. Notas sobre o gesto. Artefilosofia, Ouro Preto, n. 4, p. 9-14, jan. 2008.

AGAMBEN, Giorgio. Opus dei: arqueologia do ofício. São Paulo: Boitempo, 2013.

ARAÚJO, André Vieira de Freitas. Palestra pionerismo bibliográfico em um polímeta do séc. XVI: Conrad Gesner. Rio de Janeiro: UFRJ, 2014.

BENHABIB, Seyla. Dignity in adversity: human rights in trouble time. Cambridge: Polity press, 2011.

BUCKLAND, Michael. Redesigning library services: a manifesto. Chicago: American Library Association, 1992.

BUONOCORE, Domingo. Diccionario de bibliotecologia. 2. ed. Buenos Aires: Marymar, 1976.

CHARTIER, Roger. A ordem dos livros: leitores, autores e bibliotecas na Europa entre os séculos XIV e XVIII. 2. ed. Brasília: Ed. UnB, 1999.

COUTO, Mia. Cada homem é uma raça: contos. São Paulo: Companhia das Letras, 2013.

CRIPPA, Giulia. Conferência Nas bases da bibliografia: reflexões sobre uma longa história. Rio de Janeiro: UFRJ, 2014.

CRIPPA, Giulia. O patrimônio cultural: a cidade como documento. In: CRIPPA, Giulia; MOSTAFA, Solange Puntel (Orgs.). Ciência da Informação e Documentação. São Paulo: Alínea, 2011. 
FONSECA, Edson Nery da. A Bibliografia como Ciência: da Crítica Textual à Bibliometria. Revista Brasileira de Biblioteconomia e Documentação, São Paulo, v. 12, n. 1/2, 1979.

GOMES, Hagar Espanha. O ensino de bibliografia. Revista da Escola de Biblioteconomia da UFMG, Belo Horizonte, v. 5, n. 1, 1976.

KUHLTHAU, Carol. Seeking Meaning: a process approach to library and information services. 2. ed. Westport, CT: Libraries Unlimited, 2004.

. Inside the search process: Information seeking from the user's perspective. Journal of the American Society for Information Science, v. 42, p. 361-371, 1991.

KUHN, Thomas S. A estrutura das revoluções científicas. 10. ed. São Paulo: Perspectiva, 2007.

HABERMAS, Jürgen. Fundamentação linguística da sociologia. Lisboa: edições 70, 2010. (Obras escolhidas, 1)

HJØRLAND, Birger. Arguments for 'the bibliographical paradigm'. Some thoughts inspired by the new English edition of the UDC. Information Research, v. 12, n. 4, 2007. Disponível em:

<http://www.informationr.net/ir/12-4/colis/colis06.html>. Acesso em: 10 de setembro de 2014.

NAUDÉ, Gabriel. Advis pour dresser une bibliothèque: présenté à Monseigneur le Président de Mesmes. Paris: Chez François Targa, 1627. 164 p. Disponível em:

<http://gallica.bnf.fr/ark:/12148/bpt6k576966.r=Catalogues + de+la+biblioth\%C3\%A8que+de+Gabriel+Naud\%C3\%A9.langPT>. Acesso em: 3 nov. 2014.

OTLET, Paul. El tratado de documentación: el libro sobre el livro; teoria e prática. 2. ed. Madrid: Universidad de Murcia, 2007.

PALING, Stephen. Classification, rhetoric, and the classificatory horizon. Library Trends, v. 52, n. 3, p. 588-603, 2004.

PEIGNOT, Gabriel. Dictionnaire raisonné de bibliologie, tomo I. Paris: Chez Villier, 1802.

RANGANATHAN, Shiyali Ramamrita. Social bibliography or physical bibliography for librarians. Delhi: University of Delhi, 1952.

ROUANET, Sergio Paulo. Édipo e o anjo: itinerários freudianos em Walter Benjamin. 3. ed. Rio de Janeiro: Tempo Brasileiro, 2008. 
TENNIS, Joseph. Is there a new bibliography? Cataloging \&

Classification Quarterly, v. 49, n. 2, p. 121-126, 2011.

WITTGENSTEIN, Ludwig. Cultura e valor. Lisboa: Edições 70, 2000.

WITTGENSTEIN, Ludwig. Investigações filosóficas. 5 ed. Petrópolis: Vozes, 2008.

\section{Title}

Gesture bibliographic and modernity

\section{Abstract}

Introduction: The bibliography sharing a tensile discursive sphere, sometimes intensely textual, statistical now too. For now, in general, is put in perspective the bibliography as a linguistic performance that door can millennia of tradition, turns, twists, stagnation, which however does not start his freshness. The reverse, to becoming and ever more exciting, see the lines of Gabriel Peignot "the bibliography is the most vast and universal of all human knowledge", and Alfredo Serrai "the bibliography is the mother of all disciplines of representation writing".

Objective: This paper aims to present the dynamic tensile Bibliography and its evaluative alternations. The central intention is to weave as a matter of principle of reading the linguistic-pragmatic dimension.

Methodology: The theoretical and reconstructive approach is discussed, as of opening possibilities to instances subject to development and achievement. Understanding it as Paul Otlet, as a means, we are guided by their uses cognitive and communicative. However, we also inquire about modernity literature and its ongoing discussion questions: has finally "something bigger" as a general science of books / documents, as in Otlet? Or, you exegetical art of hermeneutics and critical? Or something else entirely? Or perhaps not so much or so little, there is a possible reflective equilibrium to be established?

Conclusions: We guide us "to" the humanistic-universal dimension involved in the bibliography, believing this to be a means for the public use of reason as propagated, but neither held by modernity. Lastly, the arguments put about a due recontextualization and a possible reconstruction of some bibliographic assumptions, aiming their emancipatory potential, once inconclusive in explaining the project (Aufklärung).

Keywords: Bibliography. Philosophy of language. Modernity.

\section{Titulo}

Gesto bibliográfico y modernidad

Resumen 
Introducción: La bibliografía compartir una esfera discursiva a la tracción, a veces intensamente textual, ahora también estadística. Por ahora, en general, se pone en perspectiva la bibliografía como una actuación lingüística esa puerta puede milenios de tradición, vueltas, giros, el estancamiento, que sin embargo no se inicia su frescura. En el reverso, para llegar a ser y cada vez más emocionante, ver las líneas de Gabriel Peignot "la bibliografía es la más amplia y universal de todo el conocimiento humano", y Alfredo Serrai "la bibliografía es la madre de todas las disciplinas de la representación escritura".

Objetivo: Este documento tiene como objetivo presentar la tracción dinámica Bibliografía y sus alternancias evaluativos. El propósito central es tejer como una cuestión de principio de la lectura la dimensión lingüístico-pragmática.

Metodología: Se discute el enfoque teórico y reconstructiva, a partir de la apertura de posibilidades a los casos sometidos al desarrollo y logro. Entendiéndo la bibliografía como Paul Otlet, como medio, nos guiamos por sus usos - cognitivos y comunicativos. Sin embargo, también nos preguntamos acerca de la literatura modernidad y sus preguntas para el debate en curso: tiene por fin "algo más grande" como una ciencia general de libros / documentos, como en Otlet? $O$ bien, arte exegética de la hermenéutica y crítica? O algo completamente distinto? O tal vez no tanto o tan poco, hay un posible equilibrio reflexivo para ser establecido?

Conclusiones: Orientamos a nosotros a la dimensión humanista universal, implicado en la bibliografía, creyendo que se trata de un medio para el uso público de la razón como propagado, pero ni en poder de la modernidad. Por último, los argumentos acerca de un debido recontextualización y una posible reconstrucción de algunos supuestos bibliográficas, con el objetivo de su potencial emancipador, una vez concluyente para explicar el proyecto (Aufklärung).

Palabras-clave: Bibliografía. Filosofía del lenguaje. Modernidad.

Recebido em: Abril de 2015

Aceito em: Julho de 2015 